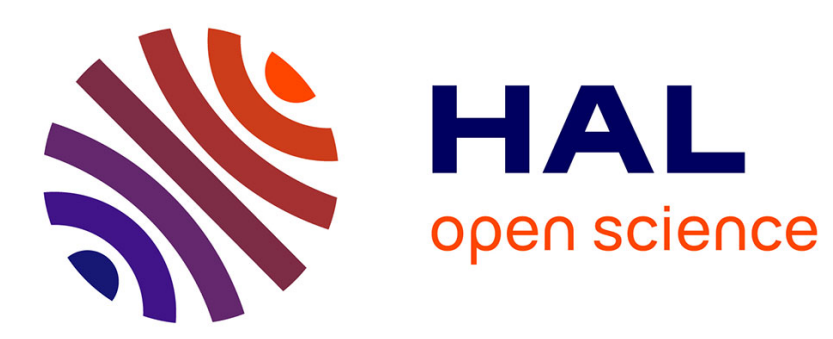

\title{
Relaxation de charge d'espace avec électrodes partiellement bloquantes et effet Maxwell Wagner : Application au nylon 66 (Hexaméthylène adipamide)
}

M. Maitrot, R. Michel, R. Madru

\section{- To cite this version:}

M. Maitrot, R. Michel, R. Madru. Relaxation de charge d'espace avec électrodes partiellement bloquantes et effet Maxwell Wagner: Application au nylon 66 (Hexaméthylène adipamide). Revue de Physique Appliquée, 1971, 6 (3), pp.369-376. 10.1051/rphysap:0197100603036900 . jpa-00243558

HAL Id: jpa-00243558

https://hal.science/jpa-00243558

Submitted on 1 Jan 1971

HAL is a multi-disciplinary open access archive for the deposit and dissemination of scientific research documents, whether they are published or not. The documents may come from teaching and research institutions in France or abroad, or from public or private research centers.
L'archive ouverte pluridisciplinaire HAL, est destinée au dépôt et à la diffusion de documents scientifiques de niveau recherche, publiés ou non, émanant des établissements d'enseignement et de recherche français ou étrangers, des laboratoires publics ou privés. 


\title{
RELAXATION DE CHARGE D'ESPACE AVEC ÉLECTRODES PARTIELLEMENT BLOQUANTES ET EFFET MAXWELL WAGNER : APPLICATION AU NYLON 66 (Hexaméthylène adipamide)
}

\author{
M. MAITROT, R. MICHEL (*) et R. MADRU \\ Laboratoire de Physique Electronique II, Faculté des Sciences de Lyon
}

(Reçu le 26 janvier 1971, révisé le 26 mars 1971)

\begin{abstract}
Résumé. - On a trouvé un effet de relaxation très net et reproductible sur le nylon [1] et on a associé aux mesures TBF de constante diélectrique des mesures de répartition de potentiel pour s'assurer qu'il s'agissait bien d'un phénomène d'électrodes; la valeur constante lorsqu'on opère successivement à différentes températures du maximum de la courbe donnant le facteur de pertes en fonction de la fréquence ferait penser à un effet Maxwell Wagner, mais une tension de quelques volts en série avec l'échantillon supprime ce phénomène de relaxation.

Comme il existe une impossibilité relative pour les porteurs de se décharger aux électrodes aux températures considérées, on a essayé d'interpréter les résultats expérimentaux, en définissant un coefficient de blocage $\rho$ suivant le modèle de Friauf [2].

On a utilisé les résultats de calculs récents pour montrer que cette hypothèse conduisait, dans le cas d'un seul type de porteurs, à un phénomène de relaxation du type de Debye, et on a pu alors interpréter le coefficient $\rho$. Les variations de la fréquence de relaxation et du maximum du facteur de pertes $\operatorname{tg} \theta$ en fonction de la tension appliquée confirment les hypothèses utilisées : on peut alors généraliser les résultats de Truckhan [3].

Pour les concentrations de porteurs telles que $L / L_{\mathrm{D}}>100$ (où $L_{\mathrm{D}}$ est la longueur de Debye) les effets de charge d'espace ne peuvent se distinguer d'une relaxation de Maxwell Wagner, tant que la tension appliquée est inférieure à $0,3 \mathrm{~V}$. Aux tensions supérieures il n'est plus possible d'utiliser un modèle basé sur la linéarisation du système d'équations différentielles générales régissant le transport des charges dans l'échantillon.
\end{abstract}

\begin{abstract}
We have found a very reliable relaxation phenomenon in nylon 66 . We were able to interpret the U. L. F. comportment of the samples by using the Friauf's theory improved by recent calculations.

When a few DC volts are superposed the relaxation phenomenon disappears in good agreement with permanent space charge theories.
\end{abstract}

Introduction. - Nous avons étudié la relaxation de charge d'espace dans le nylon 66: un certain nombre de résultats ont déjà été publiés [1], nous les compléterons plus loin. Les électrodes constituées par une couche de vernis à l'argent sont semi-bloquantes : et il semble bien que les résultats expérimentaux puissent être interprétés d'une manière satisfaisante par la théorie de Friauf. Aussi avant d'aborder la partie expérimentale de notre travail, nous rappellerons en quelques mots cette théorie, prolongée par les travaux de Beaumont et Jacobs [4], et par les calculs récents de Vandershueren [5]. En faisant apparaître dans ces derniers résultats deux parties distinctes dans l'expression de $\varepsilon^{\prime \prime}$ (où $\varepsilon^{\prime \prime}$ est la partie imaginaire de la permittivité complexe $\varepsilon^{*}=\varepsilon^{\prime}-j \varepsilon^{\prime \prime}$ ) on sera amené à identifier le coefficient $\rho$ à $2 \sigma_{\text {app }} / \sigma_{\infty}$ où $\sigma_{\mathrm{app}}$ est la conductibilité apparente en continu du matériau et $\sigma_{\infty}$ sa conductibilité pour une fréquence 'élevée. Ce coefficient découle naturellement des théories de la charge d'espace pour de faibles tensions ;

(*) R. Michel, actuellement à l'Ecole Centrale Lyonnaise. l'identification permet d'interpréter également les variations de $\varepsilon^{\prime}$. Reprenant alors la comparaison entre la théorie de Beaumont et Jacobs et un effet Maxwell Wagner, on voit que les principaux résultats coïncident.

L'identification des deux théories conduit à définir un coefficient de blocage généralement bien inférieur à 1 . D'autre part elle n'est possible que si le développement de Vandershueren est valable, c'est-à-dire pour des valeurs de $L / L_{\mathrm{D}}$ supérieures à 100 .

Nous donnerons alors les résultats expérimentaux qui confirment bien ces conclusions, et nous indiquerons les limites de validité de cette approximation.

1. Charge d'espace en l'absence de blocage total. L'accumulation de porteurs au voisinage des électrodes a fait l'objet de calculs nombreux et complexes : en continu le problème peut être résolu analytiquement si le blocage est total : mais il se complique beaucoup s'il est seulement partiel.

En alternatif les équations décrivant le comportement des porteurs doivent être linéarisées ; on admet 
qu'en chaque point la concentration $n(x)$ est de la forme $n(x)=n_{0}+n(x) \mathrm{e}^{j \omega t}$ et on admet ainsi que la densité des porteurs est en chaque point une fonction sinusoïdale du temps en phase avec la tension appliquée. Mac Donald a traité ce problème complexe avec une grande généralitẻ : un ou deux porteurs, avec ou sans recombinaison et création, mais dans l'hypothèse d'un blocage total : il montre que, dans de nombreux cas, on obtient un phénomène de relaxation du type de Debye.

Friauf introduit un coefficient de blocage $\rho$ dans les conditions aux limites : il obtient des formules complexes, et explicite la valeur de la capacité et de la résistance en parallèles équivalentes au diélectrique seulement dans un certain nombre de cas particuliers.

Beaumont et Jacobs [3] se limitent au cas d'un seul type de porteurs partiellement bloqués, sans création ni recombinaison en gardant l'hypothèse de Friauf : ce cas est précisément celui qui nous intéresse pour le nylon, où la conduction est due principalement à des porteurs positifs [10]. Beaumont et Jacobs calculent alors le courant dans le circuit extérieur du diélectrique et l'admittance équivalente à l'échantillon.

Le système d'équations définissant le problème à résoudre est le suivant :

$$
\frac{\partial n}{\partial t}=D \frac{d^{2} n}{\partial x^{2}}+\mu \frac{\partial}{\partial x}(n E)
$$

(équation de conservation des charges)

$$
\frac{\partial E}{\partial x}=\frac{e}{\varepsilon_{0}}\left(n_{0}-n_{(x)}\right)
$$

(équation de Poisson)

$$
I_{(x)}=e \cdot D \cdot \frac{\partial n}{\partial x}+e \mu n E
$$

(équation du courant local)

avec, comme conditions aux limites :

$$
\begin{gathered}
\int_{0}^{L} E \mathrm{~d} x=V \mathrm{e}^{j \omega t} \\
I_{(x=0)}=\rho \cdot \frac{D e}{L}\left(n_{(x=0)}-n_{0}\right) \\
I_{(x=L)}=-\rho \frac{D e}{L}\left(n_{(x=L)}-n_{0}\right)
\end{gathered}
$$

ces deux dernières équations pouvant servir pour la définition du paramètre de décharge $\rho ; \rho=0$, les électrodes sont parfaitement bloquantes. Les autres symboles utilisés ont leur signification classique:

$e$ : charge de l'électron;

$\mu$ : mobilité des porteurs;

$D$ : coefficient de diffusion;

$\varepsilon:$ permittivité du milieu en l'absence de charge d'espace ;

$x$ : abscisse d'un point du diélectrique mesurée selon la direction normale à ses faces;
$L:$ distance entre les faces de la lame diélectrique ; $n_{(x)}$ : concentration des porteurs au point d'abscisse;

$n_{0}$ : concentration des porteurs à l'équilibre thermique ;

$E_{(x)}$ : champ électrique au point d'abscisse $x$;

$V$ : tension électrique maximale appliquée à l'échantillon.

H. Vandershueren a montré [5] qu'il résulte des équations précédentes que l'admittance complexe de l'échantillon peut s'écrire :

$$
Y_{1}=\frac{I}{R_{0}}+j \omega C_{0}-Y
$$

où

$$
R_{0}=\frac{L}{e \mu n_{0}} \quad \text { et } \quad C_{0}=\frac{\varepsilon_{r}}{L}
$$

sont respectivement la résistance et la capacité unitaires de l'échantillon en l'absence de charge d'espace et où le terme $-Y$ représente la variation d'admittance due au phénomène de charge d'espace. Si l'on pose en outre $v=f / f_{\mathrm{c}}$ où $f$ est la fréquence de mesure et $f_{\mathrm{c}}$ la fréquence caractéristique du diélectrique $\left(f_{\mathrm{c}}=1 / 2 \pi R_{0} C_{0}\right)$

$$
A=\frac{L}{L_{\mathrm{D}}}
$$

où $L_{\mathrm{D}}$ est la longueur de Debye

$$
\delta=[1+j v]^{1 / 2} .
$$

On peut écrire

$$
\frac{1}{Y}=Z \cdot\left(1+\frac{\rho}{2}+j v A^{2} F(\delta)\right)
$$

où

$$
F(\delta)=\delta^{-2}(\delta \cot \delta-1)=\delta^{-1} \cdot \mathcal{L}(\delta)
$$

où $\mathcal{L}(\delta)$ est la fonction de Langevin. Aux très basses fréquences, lorsque $\omega \ll \omega_{\mathrm{c}}$, on peut développer la fonction $F(\delta)$ au voisinage de $v=0$; on obtient dans ce cas une expression de $Z=Z_{\mathrm{BF}}$ valable en T. B. F. et qui est de la forme

$$
Z_{\mathrm{BF}}=R_{\mathrm{BF}}+j \omega L_{\mathrm{BF}}
$$

où

$$
R_{\mathrm{BF}}=\left(\left(1+\frac{\rho}{2}\right) R_{0}+\frac{\omega^{2}}{\omega_{\mathrm{c}}^{2}} \cdot m(A) \cdot C_{0}\right)
$$

avec $\frac{\omega^{2}}{\omega_{c}^{2}} m(A)$ une fonction de $R$ qui est de valeur faible quand $A$ est grand; dans ces conditions, le terme qui dépend de la fréquence dans l'expression de $R_{\mathrm{BF}}$ devient négligeable et l'on a :

$$
R_{\mathrm{BF}}=\left(1+\frac{\rho}{2}\right) R_{0}
$$


D'autre part

$$
L_{\mathrm{BF}}=l(A) \frac{R_{0}}{\omega_{\mathrm{c}}}
$$

où

$$
l(A) \sim A=\frac{L}{L_{\mathrm{D}}} .
$$

Finalement on peut donc écrire

$$
L_{\mathrm{BF}}=\frac{L}{L_{\mathrm{D}}} \frac{R_{0}}{\omega_{\mathrm{c}}}
$$

On constate ainsi qu'en T. B. F., $R_{\mathrm{BF}}$ et $L_{\mathrm{BF}}$ sont pratiquement des constantes pour un échantillon donné, à une température donnée.

$R_{\mathrm{BF}}$ et $L_{\mathrm{BF}}$ étant les éléments composants du circuit série équivalent à $Z_{\mathrm{BF}}$, on obtient par des formules de transformation classique les éléments $R_{\mathrm{p}}, C_{\mathrm{p}} \mathrm{du}$ circuit parallèle équivalent.

$$
R_{\mathrm{p}}=\frac{\left|Z_{\mathrm{BF}}\right|^{2}}{R_{\mathrm{BF}}} \quad C_{\mathrm{p}}=-\frac{L_{\mathrm{BF}}}{\left|Z_{\mathrm{BF}}\right|^{2}}
$$

soit, en négligeant le terme en $\mathrm{m}(A)$ :

$$
\begin{aligned}
-\left(\frac{R_{\mathrm{p}}}{R_{0}}\right)^{-1} & =\frac{(1+\rho / 2)}{(1+\rho / 2)^{2}+\omega^{2}\left(l(A) \omega_{\mathrm{c}}^{-1}\right)^{2}} \\
\frac{C_{\mathrm{p}}}{C_{0}} & =\frac{l(A)}{(1+\rho / 2)^{2}+\omega^{2}\left(l(A) \omega_{\mathrm{c}}^{-1}\right)^{2}} .
\end{aligned}
$$

En fait dans le circuit global, il y a lieu de tenir compte de $R_{0}$ et $C_{0}$ montés en parallèles sur $R_{\mathrm{p}}$, $C_{\mathrm{p}}$. Si $C_{\text {eq }}$ et $R_{\text {eq }}$ sont la capacité et la résistance du circuit parallèle équivalent à l'échantillon, on a :

$$
\begin{aligned}
C_{\mathrm{eq}} & =C_{0}\left(1+\frac{C_{\mathrm{p}}}{C_{0}}\right) \\
R_{\mathrm{eq}}^{-1} & =R_{0}^{-1}\left(1-R_{\mathrm{p}}^{-1} \cdot R_{0}\right) .
\end{aligned}
$$

Les expressions de $C_{\text {eq }}$ et $R_{\text {eq }}$ peuvent s'expliciter sous la forme suivante :

$$
\begin{aligned}
C_{\mathrm{eq}} & =C_{0}\left(1+\frac{l(A)}{(1+\rho / 2)^{2}+\omega^{2}\left(l(A) \omega_{\mathrm{c}}^{-1}\right)^{2}}\right) \\
R_{\mathrm{eq}}^{-1} & =R_{0}^{-1}\left(1-\frac{1+\rho / 2}{(1+\rho / 2)^{2}+\omega^{2}\left(l(A) \omega_{\mathrm{c}}^{-1}\right)^{2}}\right) .
\end{aligned}
$$

Ces formules sont semblables à celles obtenues par Beaumont et Jacobs : elles sont valables dans le cas où $A=L / L_{\mathrm{D}}$ est grand (supérieur à $10^{2}$ ) et où $v=\omega / \omega_{\mathrm{c}}$ est petit.

L'expression de $C_{\mathrm{eq}}$ peut se ramener à la forme caractéristique d'une relaxation de type Debye soit :

$$
\varepsilon^{\prime}-\varepsilon_{\infty}=\frac{\varepsilon_{\mathrm{s}}-\varepsilon_{\infty}}{1+\omega^{2} \tau^{2}}
$$

en supposant que $\varepsilon_{\mathrm{s}} \gg \varepsilon_{\infty}$ et en posant

$$
\frac{C_{\mathrm{eq}}}{C_{0}}=\frac{\varepsilon^{\prime}}{\varepsilon_{\infty}}, \quad \varepsilon_{\mathrm{s}}=\frac{L}{L_{\mathrm{D}}} \cdot \frac{\varepsilon_{\infty}}{(1+\rho / 2)^{2}}
$$

$$
\tau=\frac{L}{L_{\mathrm{D}}} \cdot \frac{1}{\omega_{\mathrm{c}}(1+\rho / 2)}, \quad \omega_{\mathrm{c}}=\frac{1}{R_{0} C_{0}}=\frac{\sigma_{\infty}}{\varepsilon_{\infty}} .
$$

On a ainsi une expression du même type que celle obtenue par Mac Donald dans le cas d'une dissociation totale, avec $\rho=0$. Dans le cas où $\rho$ n'est pas nul, $\varepsilon_{\mathrm{s}}$ est simplement divisé par $(1+\rho / 2)^{2}$.

En procédant de même à partir de l'expression de $R_{\text {eq }}$ on peut écrire

$$
\sigma=\sigma_{\infty}\left[1-\frac{1+\rho / 2}{(1+\rho / 2)^{2}+\omega^{2}\left(l(A) \omega_{c}^{-1}\right)^{2}}\right]
$$

en posant :

$$
\frac{R_{\mathrm{eq}}}{R_{0}}=\frac{\sigma_{\infty}}{\sigma}
$$

L'expression de $\varepsilon^{\prime \prime}$ en découle

$$
\begin{aligned}
\varepsilon^{\prime \prime} & =\frac{\sigma}{\omega} \\
& =\frac{\sigma_{\infty}}{\omega}\left[\frac{(1+\rho / 2)^{2}+\omega^{2}\left(l(A) \omega_{\mathrm{c}}^{-1}\right)^{2}-(1+\rho / 2)}{(1+\rho / 2)^{2}+\omega^{2}\left(l(A) \omega_{\mathrm{c}}^{-1}\right)^{2}}\right]
\end{aligned}
$$

soit

$$
\begin{gathered}
\varepsilon^{\prime \prime}=\frac{\sigma_{\infty} \omega\left(l(A) \dot{\omega}_{c}^{-1}\right)^{2}}{(1+\rho / 2)^{2}+\omega^{2}\left(l(A) \omega_{c}^{-1}\right)^{2}}+ \\
+\frac{\sigma_{\infty}}{\omega} \frac{\rho(1+\rho / 2)}{(1+\rho / 2)^{2}+\omega^{2}\left(l(A) \omega_{c}^{-1}\right)^{2}} \\
\varepsilon^{\prime \prime}=\varepsilon_{1}^{\prime \prime}+\varepsilon_{2}^{\prime \prime} .
\end{gathered}
$$

Le premier terme $\varepsilon_{1}^{\prime \prime}$ est prépondérant quand $\rho$ est faible et $\omega$ grand. Dans ce cas $\varepsilon^{\prime \prime}$ se réduisant à $\varepsilon_{1}^{\prime \prime}$ on a

$$
\varepsilon^{\prime \prime}=\varepsilon_{\mathrm{s}} \frac{\omega \tau}{1+\omega^{2} \tau^{2}} \cdot\left(1+\frac{\rho}{2}\right)
$$

Dans ces conditions la tangente de l'angle de perte $\theta$ présente un maximum

$$
\operatorname{tg} \theta_{\max }=\frac{1}{2} \sqrt{\frac{L_{\mathrm{D}}}{L}}
$$

pour une pulsation $\omega_{\max }$

$$
\omega_{\max }=\omega_{\mathrm{c}} \sqrt{\frac{L_{\mathrm{D}}}{L}}
$$

Si l'on applique, en série avec l'échantillon, une tension continue, la valeur du paramètre de décharge $\rho$ aura tendance à croître, augmentant ainsi l'importance 
relative du terme $\varepsilon_{2}^{\prime \prime}$. Lorsque $\varepsilon_{2}^{\prime \prime}$ devient prépondérant, le maximum de $\operatorname{tg} \theta$ disparaît.

Une expression intéressante du coefficient $\rho$ peut être dérivée de sa définition. On a posé, en effet :

$$
I=\rho \frac{D}{L}\left(n_{x=L}-n_{0}\right) e
$$

soit, compte tenu de la relation d'Einstein

$$
I=\rho \frac{\left(n_{x=L}-n_{0}\right)}{n_{0}} \cdot n_{0} \mu e \frac{k T}{L e} .
$$

Les différentes théories de la charge d'espace montrent qu'au voisinage de la surface $(x=L)$, lorsque la valeur $V$ du potentiel est assez faible pour que les équations puissent être linéaires on a sensiblement [5]

$$
\frac{n_{(x=L)}-n_{0}}{n_{0}}=\frac{e V}{2 k T} .
$$

Portant cette dernière expression dans l'équation du courant, sachant que l'on a, en outre

$$
I=\sigma_{\mathrm{app}} \cdot \frac{V}{L}
$$

on en déduit

$$
\rho=2 \frac{\sigma_{\mathrm{app}}}{\sigma_{\infty}} \text { avec } \sigma_{\infty}=n_{0} \mu e .
$$

Cette même expression de $\rho$ s'obtient immédiatement lorsque l'on fait tendre $\omega$ vers zéro dans l'expression de $\sigma$ et en supposant que $\sigma_{\text {app }} / \sigma_{\infty} \ll 1$. Les résultats obtenus précédemment peuvent être retrouvés par la théorie de l'effet Maxwell Wagner appliquée à un ensemble de deux couches caractérisées respectivement par leurs épaisseurs $d_{1}, d_{2}$, leurs conductivités $\sigma_{1}, \sigma_{2}$ et leurs permittivités $\varepsilon_{1}, \varepsilon_{2}$.

L'expression de la permittivité complexe est dans ce cas de la forme [7]

$$
\varepsilon^{*}=\varepsilon_{\infty}+\frac{\varepsilon_{\mathrm{s}}-\varepsilon_{\infty}}{1+j \omega \tau}-j \frac{\sigma}{\omega}
$$

avec en particulier

$$
\sigma=\frac{\left(d_{1}+d_{2}\right) \sigma_{1} \cdot \sigma_{2}}{\sigma_{1} d_{2}+\sigma_{2} d_{1}}
$$

En basse fréquence, le dernier terme devient prépondérant, si la couche 2 a la résistivité la plus grande $\left(\sigma_{2} \ll \sigma_{1}\right)$ et est donc partiellement bloquante et si en outre l'on a :

$$
d_{2} \ll d_{1}, \quad \sigma_{1} d_{2} \gg \sigma_{2} d_{1}
$$

l'expression de la conductivité $\sigma$ se réduit,

$$
\sigma=\sigma_{2} \cdot \frac{d_{1}}{d_{2}}
$$

Dans le cas d'un phénomène de charge d'espace, $d_{2}$ est de l'ordre de la longueur de Debye $L_{\mathrm{D}}$, alors que $d_{1}$ est pratiquement égal à $L$ :

$$
\sigma=\sigma_{2} \cdot \frac{L}{L_{\mathrm{D}}} .
$$

Dans l'hypothèse où $\sigma_{1} d_{2} \gg \sigma_{2} d_{1}$; l'expression de $\varepsilon_{\mathrm{s}}$ donnée par la théorie de Maxwell peut être développée et se réduit à

$$
\varepsilon_{\mathrm{s}}=\varepsilon_{\infty} \frac{d_{1}}{d_{2}}\left(1-2 \frac{\sigma_{2}}{\sigma_{1}} \cdot \frac{d_{2}}{d_{1}}\right) .
$$

D'autre part, la théorie de la charge d'espace a conduit à l'expression (5) de $\varepsilon_{\mathrm{s}}$ qui pour de faibles valeurs de $\rho$ prend la forme

$$
\varepsilon_{\mathrm{s}}=\frac{L}{L_{\mathrm{D}}} \cdot \varepsilon_{\infty}(1-\rho) .
$$

En identifiant cette dernière expression à celle correspondant à l'effet Maxwell Wagner (15) on obtient

$$
\rho=2 \frac{\sigma_{2}}{\sigma_{1}} \cdot \frac{d_{2}}{d_{1}}
$$

soit, d'après (14) :

$$
\rho=2 \frac{\sigma_{\mathrm{app}}}{\sigma_{\infty}} \quad \text { car } \quad \sigma_{1}=\sigma_{\infty} .
$$

En conclusion, lorsque la conductibilité apparente en continu, $\sigma_{\mathrm{app}}$ est très inférieure à la conductibilité aux fréquences élevées, $\sigma_{\infty}$ (cas d'un bloquage important), l'effet de la charge d'espace se ramène à un effet Maxwell Wagner : le maximum de $\operatorname{tg} \theta$ ainsi que la fréquence correspondant à ce maximum ne dépendent que de l'épaisseur relative des deux couches et non du rapport de leurs conductibilités. Ces résultats sont à rapprocher de ceux de Trukhan : celui-ci a montré que si $L / L_{\mathrm{D}}$ était supérieur à 100 environ, l'effet de charge d'espace ne se distinguait pas d'un effet de Maxwell Wagner dans le cas d'un bloquage total.

II. Résultats expérimentaux. - 1) CONSTANTE DIÉLECTRIQUE AUX TRÈS BASSES FRÉQUENCES. - AuX fréquences comprises entre $200 \mathrm{~Hz}$ et $0,2 \mathrm{~Hz}$, la capacité de l'échantillon et les pertes ont été mesurées à l'aide d'un pont réalisé selon un principe indiqué par Schreiber [8]. Aux fréquences inférieures la dispersion $\varepsilon^{\prime}$, et l'absorption $\varepsilon_{0}$ sont déduites de la mesure du courant traversant l'échantillon et de son déphasage par rapport à la tension alternative appliquée.

Lorsqu'aucune tension continue n'est superposée à la tension alternative on observe, d'une part une augmentation de $\varepsilon^{\prime}$ quand la fréquence diminue et ce d'autant plus rapidement que la température est plus élevée, et d'autre part, on voit que la tangente de l'angle de perte $\theta$, à température constante, passe par un maximum pour une fréquence $\omega_{0}$ qui augmente avec la température; cependant la valeur maximale 
de $\operatorname{tg} \theta$ est pratiquement indépendante de la température (Fig. 1). Quand une tension continue $V_{0}$ est superposée à la tension alternative on observe une décroissance importante de $\varepsilon^{\prime}$ à mesure que $V_{0}$ augmente; cette diminution est d'autant plus marquée à une température donnée que la période de la tension alternative est plus longue et pour une période donnée que la température est plus élevée (Fig. 3).

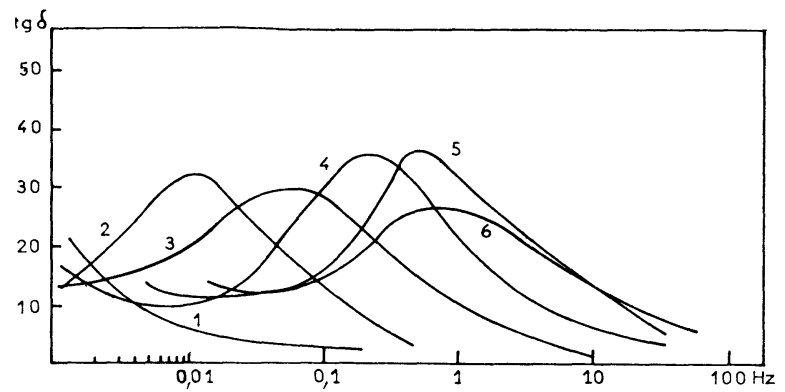

Fig. 1. - $\operatorname{tg} \theta$ en fonction de la fréquence pour différentes températures : (1) $80^{\circ} \mathrm{C}$; (2) $95^{\circ} \mathrm{C}$; (3) $105^{\circ} \mathrm{C}$; (4) $119^{\circ} \mathrm{C}$; (5) $130^{\circ} \mathrm{C}$; (6) $140^{\circ} \mathrm{C}$. Epaisseur de l'échantillon : $0,95 \mathrm{~mm}$.

L'ordonnée des courbes des figures 2 et 3 est le rapport $\varepsilon^{\prime}(V) / \varepsilon^{\prime}(0)$ où $\varepsilon^{\prime}(V)$ et $\varepsilon^{\prime}(0)$ sont respectivement les valeurs de la dispersion mesurées dans les mêmes conditions de fréquences et de température mais avec ou sans tension continue superposée. On donne également figure 3 bis la variation de $\varepsilon^{\prime}$ en fonction de la fréquence pour plusieurs températures et plusieurs tensions appliquées.

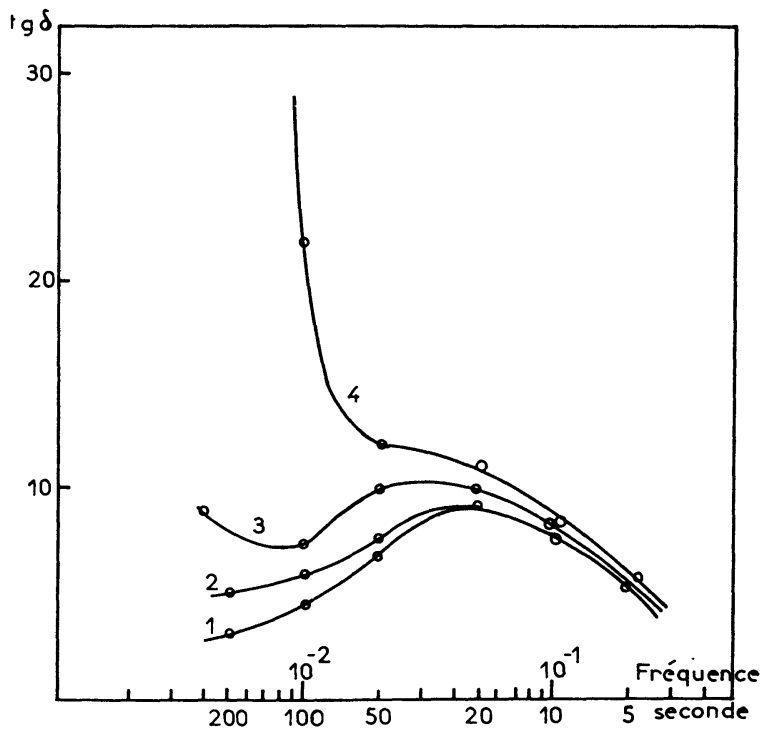

Fig. 2. - $\operatorname{tg} \theta$ en fonction de la fréquence à $105^{\circ} \mathrm{C}$ pour diverses tensions en (1) $0 \mathrm{~V}$; (2) $0,5 \mathrm{~V}$; (3) $1 \mathrm{~V}$; (4) $2 \mathrm{~V}$.

La décroissance importante de $\varepsilon^{\prime}$ quand $V$ augmente, s'accompagne d'une augmentation de $\operatorname{tg} \theta$ et de la disparition de son maximum lorsque $V$ est supérieur à $3 \mathrm{~V}$.

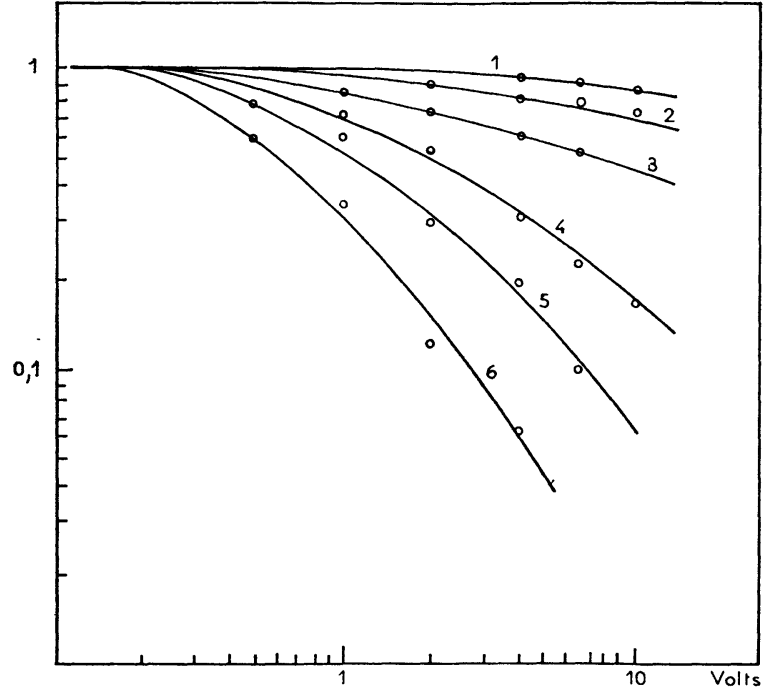

FIG. 3. - Rapport entre $\varepsilon_{(v)}^{\prime}$ à la tension $V$ et $\varepsilon_{v}^{\prime}$ pour une tension continue nulle courbe (1) période de $5 \mathrm{~s}$; (2) $10 \mathrm{~s}$; (3) $20 \mathrm{~s}$;

(4) $50 \mathrm{~s}$; (5) $100 \mathrm{~s}$; (6) $200 \mathrm{~s}$. Température $104^{\circ} \mathrm{C}$.

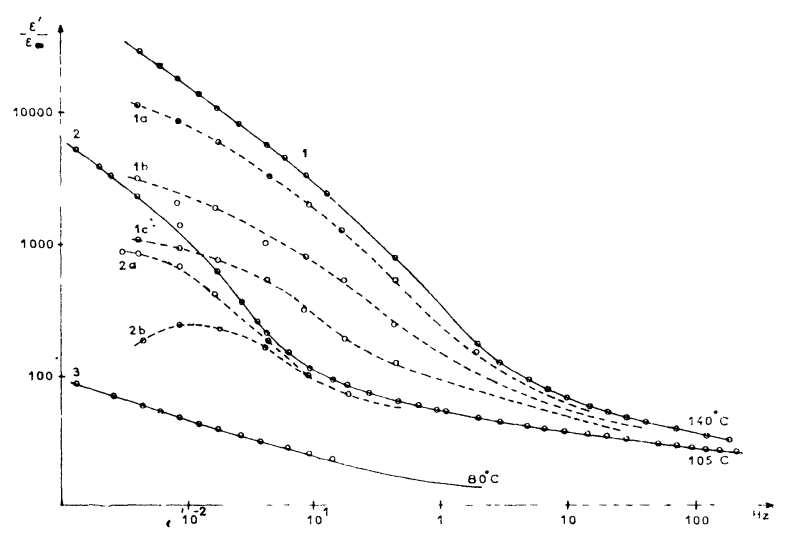

FIG. 3 bis. $-\varepsilon_{V=0}^{\prime} / \varepsilon_{\infty}^{\prime}$ et $\varepsilon_{V}^{\prime} / \varepsilon_{\infty}^{\prime}$ à diverses températures et pour diverses tensions continues superposées. Courbe $1140^{\circ} \mathrm{C}$ sans tension continue ; $1 \mathrm{a} 140^{\circ} \mathrm{C} 0,5 \mathrm{~V}$ appliqué ; $1 \mathrm{~b} 140^{\circ} \mathrm{C} 1 \mathrm{~V}$ appliqué ; $1 \mathrm{c} 140^{\circ} \mathrm{C} 2 \mathrm{~V}$ appliqués ; Courbe $2105^{\circ} \mathrm{C}$ sans tension ; $2 a 105^{\circ} \mathrm{C} 1 \mathrm{~V} ; 2 b 105^{\circ} \mathrm{C} 2 \mathrm{~V}$; Courbe (3) $80^{\circ} \mathrm{C}$, l'effet d'une tension continue superposée est presque négligeable dans la région où les mesures sont possibles.

2) RÉPARTITION DU POTENTIEL DANS L'ÉCHANTILLON. - Une étude qualitative de la répartition du potentiel à travers l'échantillon a été faite par la méthode du fil vibrant [9], [10]. On a perfectionné la méthode pour mesurer la répartition sur la tranche des échantillons étudiés en T. B. F. (épaisseur 1,5 mm).

Lorsqu'on applique une tension continue, il s'établit, dans un temps de l'ordre de la minute et qui est donc voisin des temps de relaxation précédemment déterminés une certaine répartition du potentiel à travers l'échantillon (Fig. 4).

Aux tensions faibles, inférieures à $0,3 \mathrm{~V}$, il se produit une chute très importante du potentiel au voisinage de l'anode ; quand la tension appliquée est augmentée, la répartition du potentiel tend à devenir linéaire. Ceci peut s'interpréter en considérant que la conduc- 


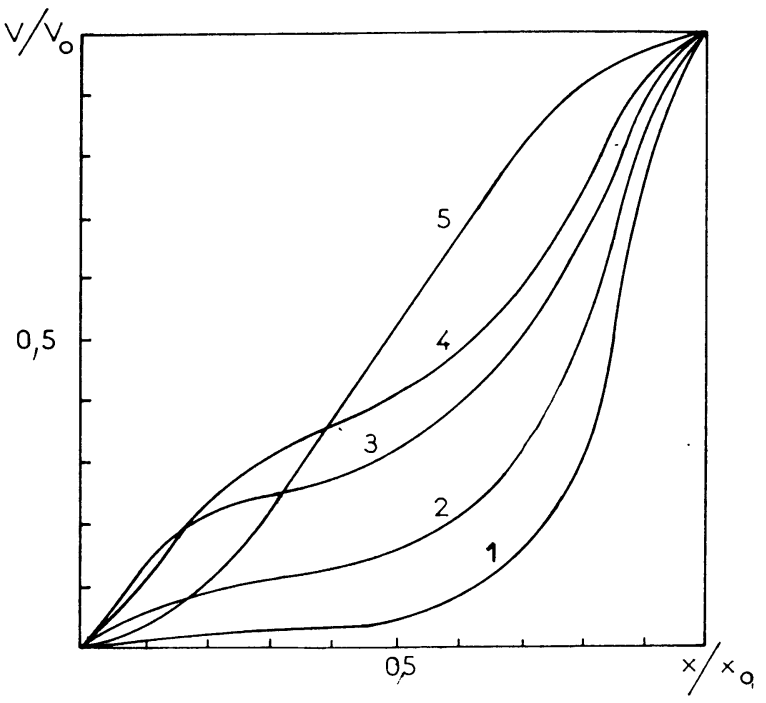

FIG. 4. - Répartition du potentiel dans le nylon mesurée par la méthode du fil vibrant : (1) $0,1 \mathrm{~V}$; (2) $0,2 \mathrm{~V}$; (3) $0,4 \mathrm{~V}$; (4) $0,8 \mathrm{~V}$; Température $140^{\circ} \mathrm{C}$.

tion dans le nylon 66 déshydraté est principalement assurée par des charges positives qui sont peut-être des protons [11]. Aux tensions faibles les contacts sont bloquants et il se forme au voisinage de l'anode une zone d'appauvrissement chargée négativement. Aux tensions plus élevées, la répartition du potentiel devient linéaire, soit que les contacts cessent d'être bloquants, et qu'il y ait transfert des charges à partir des électrodes et alors la zone d'appauvrissement précédente disparaît, soit que l'accumulation de porteurs près des électrodes devienne négligeable par tout autre processus [6]. Lorsqu'une faible tension demeure appliquée à l'échantillon très longtemps, on observe une lente évolution de la configuration précédente qui aboutit au bout d'une centaine d'heures environ, à une répartition symétrique du potentiel dans l'échantillon il se forme probablement une zone d'appauvrissement au voisinage de la cathode comme au voisinage de l'anode (Fig. 5); on sait en effet qu'une distribution symétrique de potentiel dans un échantillon bloqué correspond au cas de deux charges mobiles [9] de signe différent.

Ce dernier phénomène peut s'expliquer si l'on admet qu'il y a, en fait, dans le nylon 66 deux types de porteurs mobiles: les porteurs positifs dont le déplacement relativement rapide est responsable de la charge d'espace observée quelques minutes après application de la tension et des porteurs de charge négative, peut-être des ions $\mathrm{OH}^{-}$, de mobilité très faible dont le très lent déplacement aboutit finalement à la répartition symétrique observée au bout d'une centaine d'heures.

III. Discussion des résultats expérimentaux. VALIDITÉ DU MODĖLE PROPOSÉ. - On peut considérer que l'interprétation obtenue à partir du modèle
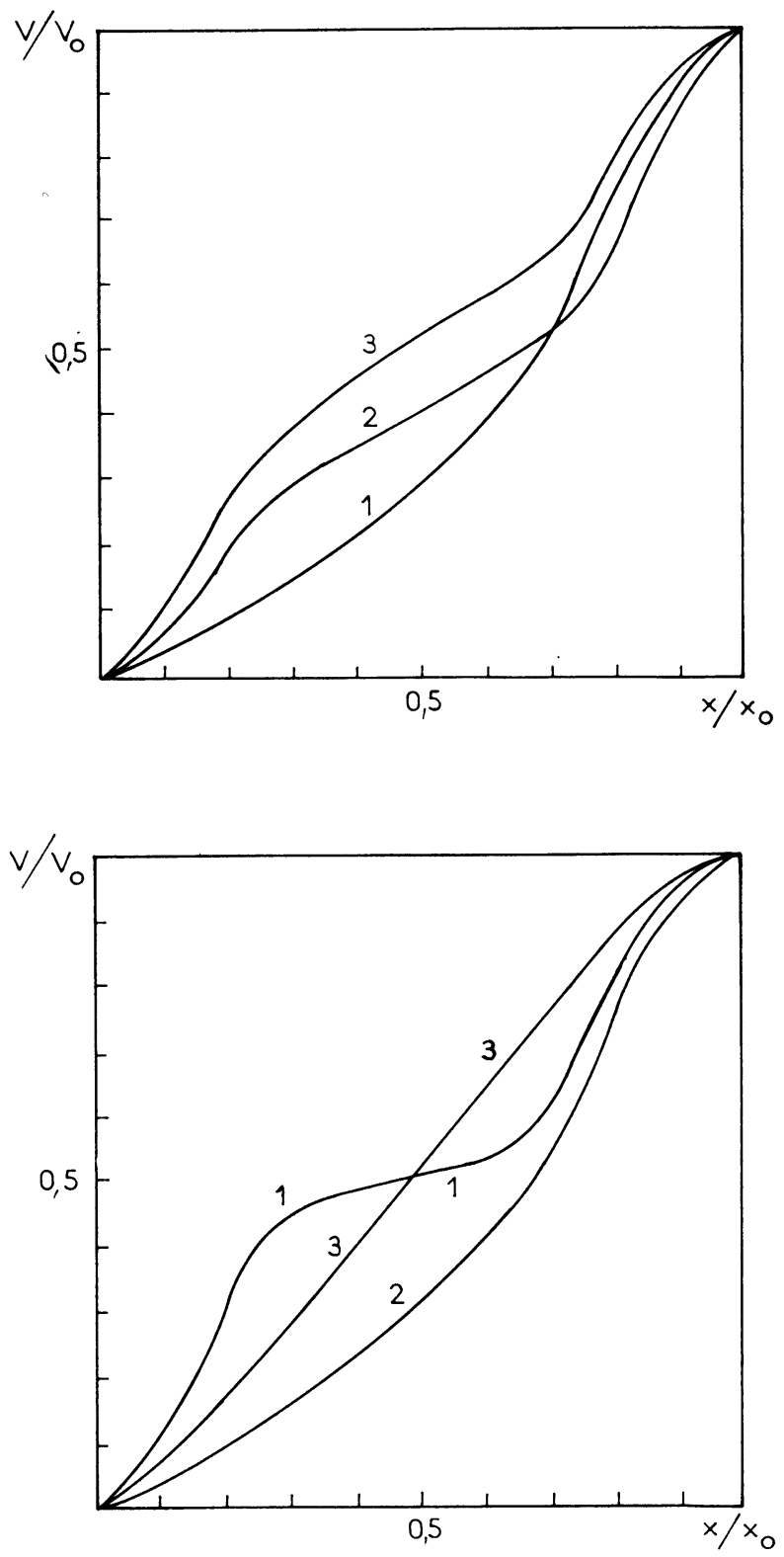

Fig. 5a. - Répartition de potentiel à l'équilibre dans le nylon sous une tension de $1,5 \mathrm{~V}$ pour diverses températures $(1) 75^{\circ} \mathrm{C}$; (2) $105^{\circ} \mathrm{C}$; (3) $140^{\circ} \mathrm{C}$. b. - Répartition de potentiel à l'équilibre à une température de $105^{\circ} \mathrm{C}$ pour diverses tensions appliquées. Courbe (1) $0,2 \mathrm{~V}$; courbe (2) $1,5 \mathrm{~V}$; courbe (3) $6 \mathrm{~V}$.

précédent est satisfaisante lorsqu'il n'y a pas de tension continue en série avec l'échantillon. En effet :

a) On peut chercher quelle est l'importance relative des deux termes de $\varepsilon^{\prime \prime}$ en posant :

$$
B=\frac{\varepsilon_{1}^{\prime \prime}}{\varepsilon_{2}^{\prime \prime}}=\frac{\omega^{2} l^{2}(A)}{\omega_{\mathrm{c}}^{2} \rho(1+\rho / 2)}
$$

et le premier terme est supérieur au second tant que

$$
\omega>\omega_{\mathrm{c}} \rho^{1 / 2} \frac{L_{\mathrm{D}}}{L}
$$

c'est-à-dire dans presque tout l'intervalle des fré- 
quences utilisées : ainsi pour la fréquence correspondant au maximum de $\operatorname{tg} \theta$, on aura :

$$
B_{\mathrm{M}}=\frac{\omega_{\mathrm{M}}^{2}}{\omega_{\mathrm{c}}^{2}} \frac{l^{2}(A)}{\rho(1+\rho / 2)}=\frac{l(A)}{\rho(1+\rho / 2)} .
$$

Mais $l(A)$ est $>100, \rho<2$; donc quand le calcul est valable, le pic de $\operatorname{tg} \theta$ est toujours visible : nous avons vu que c'est le cas dans un grand intervalle de température.

b) Les valeurs maximales de $\operatorname{tg} \theta$ sont voisines de 35 ; on en déduit donc d'après (9) que $L / L_{\mathrm{D}} \sim 5 \times 10^{3}$; ceci correspond à une longueur de Debye voisine de $2 \times 10^{-7} \mathrm{~m}$ et à une concentration en porteurs $n=3.10^{20} / \mathrm{m}^{3}$ pour $t=105^{\circ} \mathrm{C}$

$$
B_{\mathrm{M}}=\frac{5 \times 10^{3}}{\rho(1+\rho / 2)} \gg 1 \text {. }
$$

c) On peut, pour vérifier l'ordre de grandeur de $L / L_{\mathrm{D}}$ utiliser les courbes de répartition de potentiel à l'équilibre (voir référence [9] p. 71).

On a en effet

$$
-\frac{L}{L_{\mathrm{D}}} \frac{k T}{e} V_{(x)}+\left(\frac{\mathrm{d} V_{(x)}}{\mathrm{d} x}\right)^{2}=\mathrm{Cte}
$$

en admettant que la courbure de la répartition de potentiel est presque constante, dans un intervalle important d'abscisses.

On trouve $L / L_{\mathrm{D}} \simeq 4200$ pour $V=0,1 \mathrm{~V}$ et pour $V=0,2 \mathrm{~V}$ continu soit une valeur bien comparable à la précédente : cette appréciation est moins précise que la mesure de $\operatorname{tg} \theta_{\max }$.

d) Cependant on ne constate pas de pallier pour $\varepsilon^{\prime}$. Mais ceci peut s'expliquer aisément : en effet considérons le modèle de Debye pour les grandes valeurs de $\operatorname{tg} \theta$. Il y a un décalage très important entre la fréquence du maximum de $\operatorname{tg} \theta$ et la fréquence de relaxation (rapport $\left.\left(L_{\mathrm{D}} / L\right)^{1 / 2}\right)$. Le pallier de $\varepsilon^{\prime}$ ne débute lui-même qu'à une fréquence $\omega_{\mathrm{M}} L_{\mathrm{D}} / L$ soit une fréquence 5000 fois plus faible que celle du maximum de $\operatorname{tg} \theta$.

C'est ce qui rend l'étude de $\operatorname{tg} \theta$ plus facile que celle de $\varepsilon^{\prime}$. Cependant pour les plus basses fréquences $\left(10^{-3} \mathrm{~Hz}\right)$ et à $140^{\circ}$ on devrait voir le début du pallier de $\varepsilon^{\prime}$; ce pallier donnant des valeurs de $\varepsilon^{\prime} \sim 5000$ fois supérieures à $\varepsilon_{\infty}$.

Nous trouvons effectivement des valeurs de l'ordre de $5000 \varepsilon_{\infty}$ mais $\varepsilon^{\prime}$ continue à augmenter.

Nous pensons que ceci est dû aux charges négatives, moins mobiles que les charges positives (environ 100 fois moins mobiles) : ces charges sont responsables de la répartition symétrique du potentiel à l'équilibre ; on trouverait un $2^{\mathrm{e}}$ pallier dû à la relaxation de ces charges en descendant à des fréquences de l'ordre de $10^{-5} \mathrm{~Hz}$ ou $10^{-6} \mathrm{~Hz}$.

L'évolution de la capacité en fonction de la fréquence présente seulement une pente voisine de 1 à 1,5: Goffaux au contraire [13] observe deux zones de dispersion en très basse fréquence pour de faibles tensions et il n'observe pas non plus de pallier. Mais les conditions de mesure (épaisseur de l'échantillon, humidité, température) sont différentes. La variation de la capacité différentielle avec la tension est aussi différente, puisqu'il observe une augmentation, plutôt qu'une diminution brutale de la capacité différentielle dans la région des potentiels moyens.

e) La fréquence $\omega_{M}$ correspondant au maximum de $\operatorname{tg} \theta$ permet par la formule (10) le calcul de $\omega_{\mathrm{c}}$ dont on déduit $\sigma_{\infty}=\varepsilon_{\infty} \omega_{\mathrm{c}}$.

Enfin la connaissance de $\sigma_{\infty}$ et $n$ mène à la valeur de la mobilité $\mu=\sigma_{\infty} / n e$.

C'est ainsi que pour $t=105^{\circ} \mathrm{C}$ on trouve $\mu=0,6 \times 10^{-10} \mathrm{~m}^{2} / \mathrm{s}$.V. Les valeurs numériques ainsi obtenues, et qui correspondent à différentes températures de l'intervalle $80-140^{\circ} \mathrm{C}$ sont portées dans le tableau 1. De même sont portées dans ce tableau les valeurs de $\sigma_{\infty}$ mesurées à $1,5 \mathrm{kHz}$ de $\sigma_{\text {app }}$ mesurées en continu et du coefficient de bloquage $\rho$, en l'absence de tension continue superposée, et que l'on déduit de la formule $\rho=2 \sigma_{\text {app }} / \sigma_{\infty}$. L'accord entre les valeurs calculées et mesurées de $\sigma_{\infty}$ est très bon à $105^{\circ}$ et à $102^{\circ} \mathrm{C}$, il y a un écart d'un facteur 2 à $130^{\circ} \mathrm{C}$ et $140^{\circ} \mathrm{C}$.

f) Cependant la pente de $\log \varepsilon^{\prime \prime}$ en fonction de Log $\omega$ est un peu inférieure à 1 et le maximum de $\operatorname{tg} \theta$ est un peu plus large que celui d'une relaxation

TABLEAU I

\section{Température}

Fréquence de relaxation diélectrique $\omega_{\max }$

Valeur de $\sigma_{\infty}$ tirée de $\omega_{\mathrm{c}}$ $\omega_{\max }=\omega_{\mathrm{c}}\left(L_{D} / L\right)^{1 / 2}$

Valeur mesurée de $\sigma_{\infty}(1500 \mathrm{~Hz})$

Conductibilité continue $\sigma_{\mathrm{app}}$

Coefficient de bloquage sans tension superposée $=2 \sigma_{\text {app }} / \sigma_{\infty}$ $80^{\circ} \mathrm{C}$

$5 \times 10^{-3}$

(Hz)

$2 \times 10^{-12}$

$4 \times 10^{-14}$

$(\Omega \mathrm{m})^{-1}$

$4 \times 10^{-2}$ $105^{\circ}$

$8 \times 10^{-2}$

(Hz)

$3 \times 10^{-9}$

$3 \times 10^{-9}$

$6 \times 10^{-11}$

$4 \times 10^{-2}$ $120^{\circ}$

$2 \times 10^{-2}$

(Hz)

$15 \times 10^{-9}$

$12 \times 10^{-9}$

$7 \times 10^{-10}$

$10^{-1}$ $130^{\circ}$

$6 \times 10^{-1}$

(Hz)

(Hz)

$$
\begin{array}{crrr}
25 \times 10^{-9} & 6 & \times 10^{-8} \\
15 \times 10^{-9} & 30 & \times 10^{-9} \\
10^{-9} & 3 & \times 10^{-9}
\end{array}
$$

$10^{-1}$ 
de type de Debye à un seul temps de relaxation : il y aurait une faible dispersion des temps de relaxation autour d'une valeur moyenne.

- Quand il y a une tension continue superposée à la tension alternative, les résultats expérimentaux ne peuvent s'interpréter seulement par une augmentation de $\rho$. Celle-ci conduirait en effet à une réduction uniforme de $\varepsilon^{\prime}$ avec la fréquence, et, avec les valeurs numériques précédentes, on n'observerait pas de compétition entre les deux termes de $\varepsilon^{\prime \prime}$; on aurait toujours $\varepsilon_{1}^{\prime \prime} \gg \varepsilon_{2}^{\prime \prime}$. Il semble donc que cette théorie linéarisée soit insuffisante ; par contre il est possible de rapprocher la variation de la capacité différentielle avec la tension des résultats de Jacobs et Maycock $£ 5[15]$ et peut-être de résultats théoriques antérieurs [6].
Conclusion. - On a étudié la relaxation de charge d'espace dans le cas d'un seul porteur sans recombinaison, le coefficient de bloquage introduit par Friauf est toujours très faible dans les cas où l'on peut attendre une augmentation mesurable de la constante diélectrique en T. B. F. Ce coefficient de bloquage ne dépend pas de la tension appliquée d'une manière mesurable, mais reste inférieur à 1 lorsque l'on ne dépasse pas une tension de l'ordre de $0,3 \mathrm{~V}$, et dans ce cas on ne peut séparer une polarisation par charge d'espace d'un effet de Maxwell Wagner; cependant cet effet disparaît pour une tension superposée de quelques volts, conformément aux théories de la charge d'espace en continu; ceci ne serait pas obtenu pour une inhomogénéité due à la nature des couches superficielles ou à l'adsorption.

\section{Bibliographie}

[1] Michel (R.), Asch (G.), Maitrot (M.), C. R. Acad. Sci. Paris, 1970, 270, 1644-1647.

[2] Friauf (R. J.), J. Chem. Phys., 1954, 22, 1329.

[3] Trukhan (E. M.), Soviet Physics Solid State, 1963, 4, 2560.

[4] Beaumont (J. H.) et Jacobs (P. W. M.), J. Phys. Chem. Solids, 1967, 28, 657.

[5] VANDeRshueren (H.), Bulletin de la Faculté des Sciences Appliquées de l'Université de Liège, 1970.

[6] Huck (J.) et MAITrot (M.), Journal de Physique Appliquée, 1965, A 35A, 229.

[7] Van Hippel (A.), Molecular Science et Molecular engineering, p. 363, ed. Wiley.
[8] Schreiber (D. J.), J. Res. Nat. Bur. Stad., 1961, 65C, 23.

[9] Godefroy (G.), Thèse Paris, 1963.

[10] Maitrot (M.), Madru (R.), C. R. Acad. Sci., Paris, 1970, 270, 377-80.

[11] Lian Tse Yu, J. Physique, 1963, 24, 677-693.

[12] KRYZZEwSKi, SzymanSKI, SwiATEK, Journal of Polymer Science Part C No 16.

[13] Goffaux (R.), Revue Générale d'Electricité, 1966, 75, 1250-1258 ; 1967, 76, 698-704. Mem. Cl. Sci. Acad roy de Belgique, 1968, 38.

[14] JaCobs (P. W. M.) et MaYcock (J. N.), J. Chem. Phys. 1963, 39, 757. 